\title{
Difference in Cognitive Function by First Onset Age of Alcohol Induced Blackout and Its Duration
}

\author{
Eun-Jeong Min ${ }^{1}$, Sung-Gon Kim ${ }^{1,2}$, Jin-Seong Lee ${ }^{1,2}$, Bia Seo ${ }^{1}$, Woo-Young Jung ${ }^{1}$, Sung-Young Huh ${ }^{1}$, \\ Ji-Hun Park ${ }^{3}$, Chang-Hee Hong ${ }^{4}$, Hee Jung $\mathrm{Yu}^{5}$ \\ ${ }^{1}$ Department of Psychiatry, Pusan National University Yangsan Hospital, ${ }^{2}$ Department of Psychiatry, Pusan National University School of \\ Medicine, Yangsan, ${ }^{3}$ Park Ji Hun Neuropsychiatry, ${ }^{4}$ Department of Psychology, Pusan National University, ${ }^{5}$ Department of Social Welfare \& \\ Counseling, Catholic University of Pusan, Busan, Korea
}

\begin{abstract}
Objective: Alcohol-induced blackout (blackout) is a typical early symptom of cognitive impairment caused by drinking. However, the first onset age of blackout or the duration after onset of blackout has not been directly compared in previous studies. The purpose of this study was to investigate the differences in cognitive function to the first start age of blackouts and their duration.

Methods: Thirty-one male subjects were included in this study. Their age at the first blackout and the duration after the onset of blackout were investigated. Neuropsychological tests were conducted to determine their attention, memory, and executive function. Subjects were divided into three groups according to their age of the first onset blackout (group O1, < 20 years; group O2, 21-39 years; and group O3, > 40 years). Subjects were also divided into three groups by duration after the onset of blackout (P1, < 10 years; P2, 10-29 years; and P3, > 30 years). We then examined differences in neurocognitive function among these groups.

Results: $\mathrm{O} 1$ tended to have a lower memory score than $\mathrm{O} 2(F=3.28, p=0.053)$. Significant differences were observed in attention and executive function between groups P1 and P3 (Digit Span_backward: $F=6.07, p<0.05$; visual span_forward: $F=4.19, p<0.05$; executive intelligence quotient: $F=3.55, p<0.05$ ).

Conclusion: Greater memory impairment was detected in subjects having an earlier age of the first blackout. The longer the duration after the onset of blackout, the more impaired their attention and executive function skills.
\end{abstract}

KEY WORDS: Alcohol-induced blackout; Alcohol-induced disorder; Retrograde amnesia; Cognition; Addiction medicine.

\section{INTRODUCTION}

It is well known that alcohol use has a negative effect on cognitive function. Considerable evidence suggests that chronic alcohol use reduces the volume or activity of the hippocampus as well as the frontal lobe, leading to impairment in a wide range of cognitive functions, especially memory and executive functions [1-5]. An alco-

Received: December 26, 2018 / Revised: February 26, 2019

Accepted: May 1, 2019

Address for correspondence: Sung-Gon Kim

Department of Psychiatry, Pusan National University Yangsan

Hospital, Pusan National University School of Medicine, 20

Geumo-ro, Mulgeum-eup, Yangsan 50612, Korea

E-mail: sungkim@pusan.ac.kr

ORCID: https://orcid.org/0000-0001-7566-941X

*The findings from this study were presented at 16 th International Society of Addiction Medicine Annual Meeting, October 2-6, 2014, Yokohama, Japan. hol-induced blackout (hereinafter called a blackout) is a typical early symptom of cognitive impairment caused by drinking. A blackout has been thought to be an essential early warning sign of problematic drinking [6].

A great majority of alcoholics experience blackouts during the early phase of their addiction [7]. Even in young social drinkers, those who experience blackout are characterized by more days of drinking, higher frequency of heavy drinking, and greater number of drinks per day [8]. A blackout is defined as memory loss from a drinking episode without the loss of consciousness $[8,9]$. It can be classified as being en bloc or fragmentary depending on all or partial amnesia of drinking episodes and the extent of alcohol-induced memory loss [10]. Blackout is thought to result from failure to transfer information from short-term memory into long-term memory [11].

A comparison between individuals with and without a

(ㄷ) This is an Open-Access article distributed under the terms of the Creative Commons Attribution Non-Commercial License (http://creativecommons.org/licenses/by-nc/4.0) which permits unrestricted non-commercial use, distribution, and reproduction in any medium, provided the original work is properly cited. 
history of blackout has revealed no significant difference in memory tasks while sober. However, after alcohol consumption, individuals with a history of blackout showed poorer contextual memory compared to their counterparts $[12,13]$. Young adults with a history of blackout differed in contextual memory-related brain activation during alcohol session, but not during sober session [14]. More recent studies have indicated that binge drinking affects frontal lobe neurochemistry, especially in those who have experienced blackout [15]. Chitty et al. [16] have found that reduced hippocampal quantification of glutathione is associated with blackout presence/severity. This result upholds the idea that the hippocampus plays a role in the continuum of alcohol-induced memory impairment.

Effect of blackout on cognitive impairment, including memory, is common in young adults. It is associated with alcohol-related negative consequences $[17,18]$. In other words, this effect can be regarded as an important indicator that emerges relatively early. It is obviously connected to alcohol related problems. Previous researches have focused on the prevalence and predictors of blackout, consequences of blackout, and potential cognitive and neurobiological mechanisms [19]. However, studies on the first onset age of blackout and the duration after onset of blackout are very rare. Therefore, the purpose of this study was to investigate the differences in cognitive function to the first start age of blackouts and their duration.

\section{METHODS}

\section{Subjects}

Thirty-one male subjects were included in this study. This study retrospectively reviewed existing data and test results. Among subjects who visited Pusan National University Yangsan Hospital and Busan Community Addiction Management Center between March 2011 and July 2013, subjects who had a history of blackout with results available for neuropsychological tests were included in this study. Subjects who had alcohol-related dementia and intelligence quotient (IQ) 70 or less were excluded. During interview and neuropsychological tests, subjects were abstinent for at least one week. This study was approved by the Institutional Review Board of Pusan National University Yangsan Hospital (05-2016-015). Informed consent was obtained from each subject.

\section{Measures}

\section{Alcohol use questionnaire}

An alcohol use questionnaire comprising questions about the first onset age of blackout and duration after onset of blackout as well as demographic information was administered.

\section{Neurocognitive function tests}

Intelligence was assessed using the Korean Wechsler Adult Intelligence Scale-Revised (K-WAIS) [20]. Attention was assessed using the K-WAIS Digit Span subtest and visual span. Memory was assessed using the Rey-Kim memory test (RKMT) [21]. RKMT was a standardized version of the Auditory Verbal Learning Test and the Complex Figure Test developed by Andre Rey [22,23]. Executive functions were assessed using Kim's Frontal-Executive Function Neuropsychological Test (K-FENT) [24]. K-FENT consists of Stroop test, Fluency test, and Rey-Kim verbal memory retrieval session. All neurocognitive test scores were age-scaled and transformed to yield a mean \pm standard deviation of $100 \pm 15$ or $10 \pm 3$.

\section{Statistical Analyses}

We divided subjects into three groups according to the first onset age of blackout as well as the duration after onset of blackout. In previous studies, more than $50 \%$ of college students reported that they experienced blackouts [19]. Adolescents and emerging young adults often engage in a pattern of high intensity or binge drinking [25]. Those with a first subjective intoxication before age 14 years were more likely to develop alcohol dependence compared with those who had a first subjective intoxication at or after age 21 years [26]. Considering these results, subjects of the present study were divided into three groups according to the first onset age of blackout (group O1, < 20 years; group O2, 21-39 years; and group O3, $>40$ years). Since it was difficult to find references or definitions for the classification of groups according to the duration after onset of blackout, we arbitrarily divided durations into 10 years (group P1, < 10 years; group P2, 10 - 29 years; group P3, > 30 years). In group $\mathrm{P} 1$, seven subjects with an initial onset age of a first blackout at 40 years or older were excluded. This step was taken because when comparing 'the group of under 40 years old' with 'the group of over 40 years old', it was difficult to 
assume homogeneity within a group in terms of demographics or alcohol-related characteristics. Thus, 24 subjects were included in the analysis according to the duration after onset of blackout.

Three group differences were assessed with analyses of variance. Statistically, homogeneity of variance was assumed. Post-hoc analyses were conducted using Scheffé's method. The criterion for statistical significance in all tests was set at $p<0.05$. All data were analyzed using statistical package SPSS version 21.0 for Windows (IBM Corp., Armonk, NY, USA).

\section{RESULTS}

Demographic and alcohol use characteristics of subjects are shown in Table 1. No significant differences were found in neurocognitive function according to the first onset age of blackout (Table 2). However, group $\mathrm{O} 1$ tended to have lower memory score than group O2 $(F=3.28, p=$ 0.053) (Fig. 1). Table 3 shows difference in neurocognitive function by the duration after onset of blackout. Significant differences were observed in attention and executive functions between groups P1 and P3 (Digit Span_backward: $F=6.07, p<0.05$; visual span_forward:

Table 1. Demographic and clinical characteristics $(n=31)$

\begin{tabular}{ll}
\multicolumn{1}{c}{ Characteristic } & \multicolumn{1}{c}{ Data } \\
\hline Age $(\mathrm{yr})$ & $48.45 \pm 14.05$ \\
Age of the first drinking $(\mathrm{yr})$ & $18.52 \pm 8.69$ \\
Drinking duration $(\mathrm{yr})$ & $28.94 \pm 15.58$ \\
Age of the first blackout $(\mathrm{yr})$ & $32.42 \pm 14.81$ \\
Blackout duration $(\mathrm{yr})$ & $16.06 \pm 13.92$ \\
Intelligence quotient & $110.61 \pm 11.32$ \\
\hline
\end{tabular}

Values are presented as mean \pm standard deviation. $\digamma=4.19, p<0.05$; executive IQ: $F=3.55, p<0.05)$

(Figs. 2, 3).

\section{DISCUSSION}

The purpose of this study was to investigate the differences in cognitive function to the first start age of blackouts and their duration. In previous studies, individuals with a history of blackout were more vulnerable to alcohol's effects on cognitive function and brain activations than individuals without a history of blackout [12-15]. However, research on the relationship between cognitive function and the duration after onset of blackout is limited.

The presence of a blackout indicates that the drinker has reached a level of intoxication consistent with significant impairments in a variety of brain regions beyond

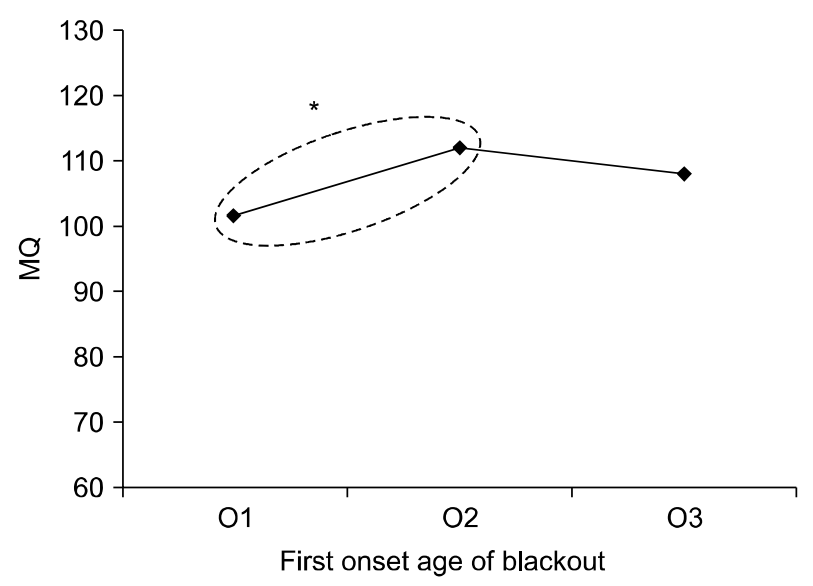

Fig. 1. Differences in memory by the first onset age of blackout. $M Q$, memory quotient; $\mathrm{O} 1, \leq 20$ years old; O2, 21 -39 years old; O3, $\geq 40$ years old. $* p<0.05$.

Table 2. Comparison of memory quotient by the first onset age of blackout

\begin{tabular}{lccccc}
\hline & O1 $(\mathrm{n}=9)$ & O2 $(\mathrm{n}=13)$ & O3 $(\mathrm{n}=9)$ & $F$ & Scheffé \\
\hline Age $(\mathrm{yr})$ & $38.89 \pm 14.98$ & $48.08 \pm 13.24$ & $58.56 \pm 5.86$ & 5.85 & 0.008 \\
Education $(\mathrm{yr})$ & $12.33 \pm 3.39$ & $12.69 \pm 3.43$ & $12.22 \pm 2.49$ & 0.07 & 0.94 \\
IQ & $108.44 \pm 10.31$ & $110.00 \pm 12.56$ & $113.67 \pm 11.00$ & 0.50 & 0.615 \\
Digit Span_forward & $7.22 \pm 1.30$ & $6.77 \pm 1.30$ & $6.78 \pm 2.11$ & 0.26 & 0.773 \\
Digit Span_backward & $5.56 \pm 1.51$ & $4.15 \pm 1.63$ & $5.00 \pm 1.50$ & 2.25 & 0.124 \\
Visual span_forward & $7.00 \pm 2.45$ & $9.33 \pm 3.00$ & $9.00 \pm 3.08$ & 1.53 & 0.236 \\
MQ & $101.56 \pm 9.40$ & $111.92 \pm 10.85$ & $108.00 \pm 6.38$ & 3.28 & 0.053 \\
Executive IQ & $103.44 \pm 14.55$ & $105.15 \pm 17.72$ & $106.11 \pm 19.25$ & 0.06 & 0.947 \\
\hline
\end{tabular}

Values are presented as mean \pm standard deviation.

$\mathrm{O} 1, \leq 20$ years old; O2, $21-39$ years old; O3, $\geq 40$ years old; IQ, intelligence quotient; $M Q$, memory quotient.

$* p<0.05$. 
Table 3. Comparison of Groups by period of blackout

\begin{tabular}{lccrrcc}
\hline & $\mathrm{P} 1(\mathrm{n}=9)$ & $\mathrm{P} 2(\mathrm{n}=8)$ & $\mathrm{P} 3(\mathrm{n}=7)$ & \multicolumn{1}{c}{$F$} & $p$ value & Scheffé \\
\hline Age $(\mathrm{yr})$ & $33.89 \pm 10.30$ & $42.88 \pm 8.25$ & $60.71 \pm 5.62$ & 19.92 & 0.000 & $\mathrm{P} 1<\mathrm{P} 3^{*}$ \\
Education (yr) & $14.89 \pm 1.76$ & $12.88 \pm 3.44$ & $9.57 \pm 2.07$ & 8.80 & 0.002 & $\mathrm{P} 1>\mathrm{P} 3^{*}$ \\
IQ & $111.67 \pm 11.54$ & $106.63 \pm 13.69$ & $111.43 \pm 8.72$ & 0.48 & 0.624 & \\
Digit Span_forward & $8.00 \pm 1.23$ & $6.63 \pm 1.30$ & $6.57 \pm 1.13$ & 3.67 & 0.043 & \\
Digit Span_backward & $6.00 \pm 1.58$ & $4.75 \pm 1.75$ & $3.43 \pm 0.79$ & 6.07 & 0.008 & $\mathrm{P} 1>\mathrm{P} 3^{*}$ \\
Visual span_forward & $10.67 \pm 2.88$ & $8.38 \pm 2.20$ & $6.71 \pm 2.36$ & 4.19 & 0.032 & $\mathrm{P} 1>\mathrm{P} 3^{*}$ \\
MQ & $111.78 \pm 9.54$ & $104.88 \pm 13.87$ & $106.71 \pm 9.00$ & 0.90 & 0.424 & \\
Executive IQ & $114.00 \pm 7.73$ & $106.50 \pm 15.85$ & $94.43 \pm 19.31$ & 3.55 & 0.047 & $\mathrm{P} 1>\mathrm{P} 3^{*}$ \\
\hline
\end{tabular}

Values are presented as mean \pm standard deviation.

$\mathrm{P} 1, \leq 10$ years period; $\mathrm{P} 2,11-29$ years period; $\mathrm{P} 3, \geq 30$ years period; IQ, intelligence quotient; $M Q$, memory quotient. $* p<0.05$.

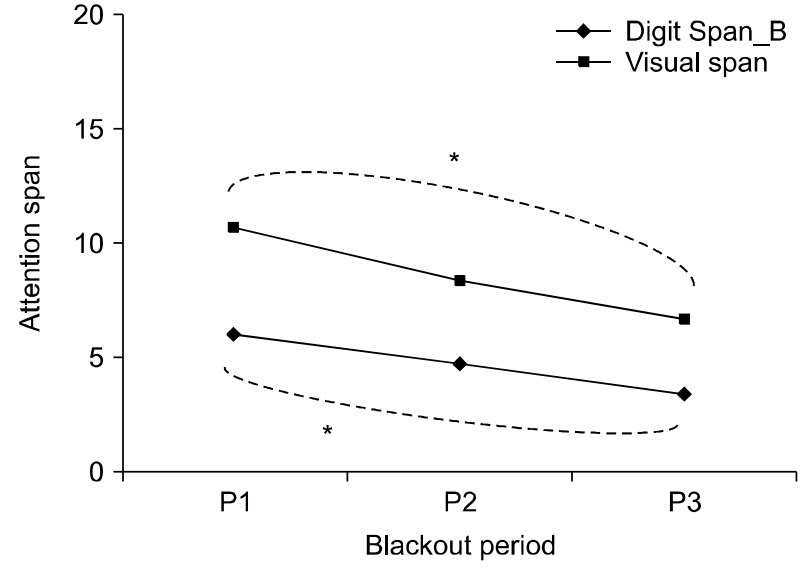

Fig. 2. Differences in attention by duration after onset age of blackout. Digit Span_B, Digit span_backward; Visual span, visual span_forward; $\mathrm{P} 1, \leq 10$ years period; $\mathrm{P} 2,11-29$ years period; $\mathrm{P} 3, \geq 30$ years period.

$* p<0.05$.

temporal lobe memory areas, including frontal lobe regions involved in attention, impulse control, and decision making $[14,27,28]$. Thus, blackout might be a better predictor of other alcohol-related outcomes such as injuries than traditional quantity/frequency measures of consumption. Hingson et al. [29] have suggested that an affirmative answer to a single question about blackouts can predict the likelihood that subjects will report a wide range of alcohol-related harmful effects better than other alcohol consumption measures.

To the best of our knowledge, the purpose of this study was to investigate the differences in cognitive function to the first start age of blackouts and their duration. However, this study has some limitations. First, definitions of the first onset age and duration after onset of blackout are somewhat vague. In addition, it was difficult to find

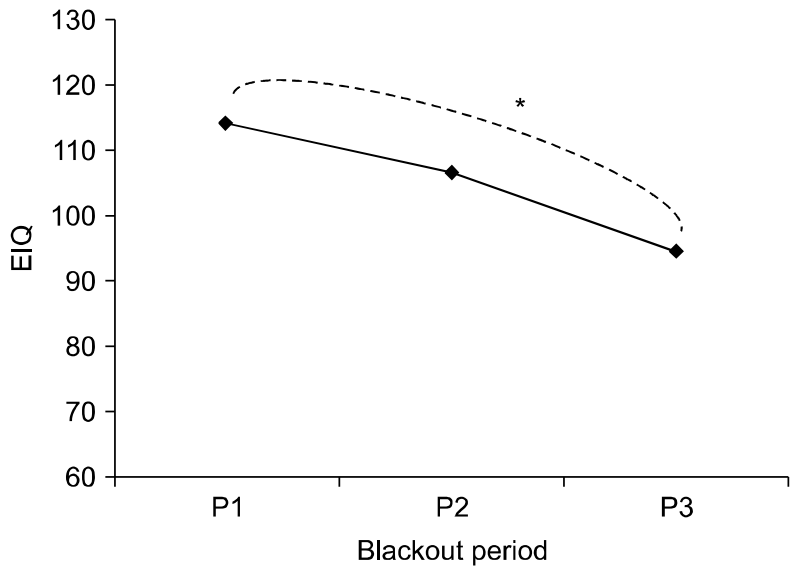

Fig. 3. Differences in executive function by duration after onset age of blackout.

EIQ, executive intelligence quotient; $\mathrm{P} 1, \leq 10$ years period; $P 2,11-$ 29 years period; $\mathrm{P} 3, \geq 30$ years period.

$* p<0.05$.

references or definitions for the classification of groups according to the duration after onset of blackout. Thus, we arbitrarily divided durations into 10 years. In addition, the duration after onset of blackout might have correlation with the duration of drinking which also can be an important factor affecting cognitive function. It is also related to frequency of blackout, frequency of alcohol consumption, frequency of alcohol use, and drinking characteristics. Second, there are some limitations in terms of method. It might be difficult to clarify whether current cognitive function problems were related to blackout or alcohol because samples of alcoholics without blackout were not included. In addition, cognitive function before blackout was not evaluated. Moreover, in this study, there were significant differences in age and education status among groups. Thus, further studies that 
control age and educational status of subjects are needed. In addition, this was a cross-sectional study. It had a limitation in the self-report method of blackout. Another limitation was that its sample size was small. Thus, there were limitations in understanding various relationships between blackout characteristics and neurocognitive function. While reviewing studies, we found an interesting study showing that the right hippocampus was significantly smaller in adult alcoholic patients with adolescent-onset use compared to that in controls or the late-onset group [4]. In the future, it will be necessary to study difference in cognitive function by the first onset age and duration after onset of a blackout. Although this study has several limitations, this study is an important preliminary study on the progress of blackout and cognitive function. Further studies accounting for these limitations will be needed.

\section{Acknowledgments}

This study was supported by Research Institute for Convergence of Biomedical Science and Technology (302017-007), Pusan National University Yangsan Hospital, Korea.

\section{- Conflicts of Interest}

No potential conflict of interest relevant to this article was reported.

\section{Author Contributions}

Conceptualization: Sung-Gon Kim. Data acquisition: Eun-Jeong Min, Sung-Gon Kim, Bia Seo, Ji-Hun Park. Formal analysis: Eun-Jeong Min, Sung-Gon Kim, Bia Seo, Woo-Young Jung, Chang-Hee Hong, Hee Jung Yu. Funding: Sung-Gon Kim. Supervision: Sung-Gon Kim. Writing-original draft: Eun-Jeong Min, Jin-Seong Lee, Bia Seo. Writing-review \& editing: Eun-Jeong Min, Sung-Gon Kim, Sung-Young Huh.

\section{ORCID}

Eun-Jeong Min Sung-Gon Kim Jin-Seong Lee Bia Seo Woo-Young Jung Sung-Young Huh Ji-Hun Park https://orcid.org/0000-0002-5474-4529 https://orcid.org/0000-0001-7566-941X https://orcid.org/0000-0001-8788-7596 https://orcid.org/0000-0002-6426-8378 https://orcid.org/0000-0003-2589-3784 https://orcid.org/0000-0001-7617-9375 https://orcid.org/0000-0002-0689-4485
Chang-Hee Hong

https://orcid.org/0000-0002-0037-0568

Hee Jung Yu

https://orcid.org/0000-0002-7128-9441

\section{REFERENCES}

1. Kril JJ, Halliday GM. Brain shrinkage in alcoholics: a decade on and what have we learned? Prog Neurobiol 1999;58:381387.

2. Moselhy HF, Georgiou G, Kahn A. Frontal lobe changes in alcoholism: a review of the literature. Alcohol Alcohol 2001; 36:357-368.

3. Beresford TP, Arciniegas DB, Alfers J, Clapp L, Martin B, Du Y, et al. Hippocampus volume loss due to chronic heavy drinking. Alcohol Clin Exp Res 2006;30:1866-1870.

4. Ozsoy S, Durak AC, Esel E. Hippocampa/ volumes and cognitive functions in adult alcoholic patients with adolescent-onset. A/cohol 2013;47:9-14.

5. Soares-Simi SL, Pastrello DM, Ferreira ZS, Yonamine M, Marcourakis T, Scavone C, et al. Changes in CREB activation in the prefrontal cortex and hippocampus blunt ethanol-induced behavioral sensitization in adolescent mice. Front Integr Neurosci 2013;7:94.

6. Lee H, Roh S, Kim DJ. Alcohol-induced blackout. Int J Environ Res Public Health 2009;6:2783-2792.

7. Fields R. Drugs and alcohol in perspective. Dubuque:Brown Publishers; 1992.

8. Jennison KM, Johnson KA. Drinking-induced blackouts among young adults: results from a national longitudinal study. Int J Addict 1994;29:23-51.

9. Rose ME, Grant JE. A/cohol-induced blackout. Phenomenology, biological basis, and gender differences. J Addict Med 2010; 4:61-73.

10. Goodwin DW, Crane JB, Guze SB. Phenomenological aspects of the alcoholic "blackout". Br I Psychiatry 1969;115: 1033-1038.

11. White AM. What happened? A/cohol, memory blackouts, and the brain. Alcohol Res Health 2003;27:186-196.

12. Hartzler B, Fromme K. Fragmentary blackouts: their etiology and effect on alcohol expectancies. Alcohol Clin Exp Res 2003;27:628-637.

13. Wetherill RR, Fromme K. Acute alcohol effects on narrative recall and contextual memory: an examination of fragmentary blackouts. Addict Behav 2011;36:886-889.

14. Wetherill RR, Schnyer DM, Fromme K. Acute alcohol effects on contextual memory BOLD response: differences based on fragmentary blackout history. Alcohol Clin Exp Res 2012;36:1108-1115.

15. Silveri MM, Cohen-Gilbert J, Crowley DJ, Rosso IM, Jensen JE, Sneider JT. Altered anterior cingulate neurochemistry in emerging adult binge drinkers with a history of alcohol-induced blackouts. Alcohol Clin Exp Res 2014;38:969-979.

16. Chitty KM, Lagopoulos J, Hickie IB, Hermens DF. The impact of alcohol and tobacco use on in vivo glutathione in youth with bipolar disorder: an exploratory study. J Psychiatr Res 
2014;55:59-67.

17. Barnett NP, Clerkin EM, Wood M, Monti PM, O'Leary Tevyaw $\mathrm{T}$, Corriveau D, et al. Description and predictors of positive and negative alcohol-related consequences in the first year of college. J Stud A/cohol Drugs 2014;75:103-114.

18. White AM, Jamieson-Drake DW, Swartzwelder HS. Prevalence and correlates of alcohol-induced blackouts among college students: results of an e-mail survey. J Am Coll Health 2002;51:117-119, 122-131.

19. Wetherill RR, Fromme K. A/cohol-induced blackouts: a review of recent clinical research with practical implications and recommendations for future studies. Alcohol Clin Exp Res 2016;40:922-935.

20. Yum TH, Park YS, Oh KJ, Kim JK, Lee YH. Korea-Wechsler Adult Intelligence Scale-Revised. Seoul:Guidance Korea;1992.

21. Kim HG. Rey-Kim memory test. Daegu:Neuropsychology Press;2006.

22. Rey A. [The psychological examination in cases of traumatic encepholopathy. Problems]. Arch Psychol 1941;28:215-285. French.

23. Rey A. L'examen clinique en psychologie. Paris:Press
Universitaires de France;1964.

24. Kim HG. Kims Frontal-executive Function Neuropsychological Test. Daegu:Neuropsychology Press;2004.

25. Patrick ME, Schulenberg JE, Martz ME, Maggs JL, O’Malley PM, Johnston LD. Extreme binge drinking among 12th-grade students in the United States: prevalence and predictors. JAMA Pediatr 2013;167:1019-1025.

26. Hingson RW, Heeren T, Winter MR. Age at drinking onset and alcohol dependence: age at onset, duration, and severity. Arch Pediatr Adolesc Med 2006; 160:739-746.

27. Bjork JM, Gilman JM. The effects of acute alcohol administration on the human brain: insights from neuroimaging. Neuropharmacology 2014;84:101-110.

28. Magrys SA, Olmstead MC. A/cohol intoxication alters cognitive skills mediated by frontal and temporal brain regions. Brain Cogn 2014;85:271-276.

29. Hingson R, Zha W, Simons-Morton B, White A. Alcohol-induced blackouts as predictors of other drinking related harms among emerging young adults. Alcohol Clin Exp Res 2016;40:776-784. 